\title{
Considerações sobre a clínica na atualidade: psiquiatria, psicanálise e saúde mental*
}

\author{
Marcio de Vasconcellos Pinheiro
}

O autor trabalhou intensamente durante muitos anos como psiquiatra e psicanalista no Brasil e nos Estados Unidos, num período de quarenta e cinco anos. Ele relata suas experiências nesses dois países, apontando semelhanças e diferenças no trabalho clínico durante esses anos, bem como para as tendências nesses países em épocas diferentes. Conclui fazendo uma previsão sobre o futuro do trabalho clínico nos EUA e Brasil.

Palavras-chave: Brasil, Estados Unidos, trabalho clínico, psiquiatria

* Trabalho apresentado no III Encontro de Psiquiatria e Saúde Mental, na Clínica Harmonia, Patos de Minas, 30/11/02. 


\begin{tabular}{llllll|l}
$R$ & $E$ & $V$ & I & S & T A & \\
LATIN O A M E RIC AN A & \\
DE PSICO PATO LOGIA & \\
F U N D A M E N T A L & \\
ano VIII, n. 3 , set/ 2005 & \\
\hline
\end{tabular}

Quando pensei em falar sobre este tema achei que havia chegado a hora de fazer um inventário sobre as minhas experiências na psiquiatria, psicanálise e saúde mental nos últimos quarenta anos, desde o início de minha residência em psiquiatria em 1961. Para melhor colocar este tema, e melhor situar meus pontos de vista, farei uma breve retrospectiva sobre a psiquiatria e a psicanálise com as quais convivi.

Em 1961, eu fazia clínica médica nos Estados Unidos quando resolvi mudar de rumo e ingressar na psiquiatria. Ao chegar ao Instituto de Psiquiatria da Universidade de Maryland, depois de ser apresentado aos colegas, professores e supervisores sob o comando do Professor Eugene Brody, logo comecei a receber pacientes para tratar. No estilo pragmático norte-americano, em poucas semanas eu estava fazendo "psicoterapia psicanalítica” com dez pacientes, sob a orientação de dois supervisores. Essa psicoterapia era feita com cada paciente pelo período de uma hora, três vezes por semana, independentemente dos diagnósticos. Eu participava também das reuniões clínicas, seminários e conferências semanais.

No prontuário de cada um dos meus casos, depois de firmar um diagnóstico psiquiátrico, eu tinha de fazer uma formulação psicodinâmica, isto é, fazer um diagnóstico clínico de acordo com o DSM-I e uma formulação psicanalítica com a dimensão estrutural (ego, id, superego), topográfica (inconsciente, pré-consciente, consciente) e genética (histórica) (Cameron, 1963). O uso da medicação psiquiátrica era desencorajado e até considerado evidência de uma psicoterapia não muito bem-feita. Acreditava-se que, para fazer psicoterapia com esquizofrênicos, por exemplo, os melhores terapeutas eram os que estavam próximos à desordem, com uma sensibilidade especial, de preferência uma esquizoidia criativa.

Naquele tempo, nos Estados Unidos, a psicanálise tinha muito prestígio, especialmente no mundo acadêmico. Ela influenciava muito a psiquiatria e tanto o professor Brody, como a maioria dos professores e supervisores, eram psicanalistas. Contudo, apesar da 
forte influência da psicanálise, o ambiente do Instituto de Psiquiatria era eclético com muitas pesquisas em todas as direções. Havia desde pesquisas biológicas até a presença de um filósofo na equipe, cuja função era simplesmente a de trocar idéias com os residentes.

O tratamento de cada caso se desenvolvia dentro de uma equipe multidisciplinar. Os pacientes podiam ficar internados um tempo longo. Não havia programas residenciais e de convivência na comunidade, além dos consultórios e de poucas clínicas públicas de saúde mental. Os pacientes com alta hospitalar sempre voltavam para a família. Os hospitais, especialmente os públicos, estavam superlotados. Eram os famosos manicômios.

Nesse período, a psicanálise era uma grande esperança da psiquiatria. Por exemplo, no hospital Chestnut Lodge, que ficava muito próximo a Washington, D.C., o paciente ficava internado muitos anos fazendo quase exclusivamente uma intensa psicoterapia psicanalítica individual, cinco vezes por semana. Além do psicanalista, o paciente tinha um médico “administrador clínico” para fazer decisões administrativas, evitando assim que essas decisões contaminassem a psicoterapia. Harry Stack Sullivan (1953, 1954), Frieda Fromm-Reichman (1950, 1959, apud Horstein, 2000), Harold Searles (1965), Otto Allen Will Jr (1967), Clarence Schulz $(1992,1969)$ entre outros psicanalistas, trabalhavam nesse hospital. O livro Nunca lhe prometi um jardim de rosas (Green, 1964) foi escrito por uma paciente tratada no hospital pela dra. Frieda Fromm-Reichman.

Sempre fui mais interessado no atendimento psicoterapêutico e psicanalítico do que nos atendimentos medicamentosos das doenças mentais. Mas lembro-me até hoje de uma jovem esquizofrênica de quem tratei sem remédios por um ano, sem grandes resultados. No fim desse ano eu a transferi para um outro residente que lhe deu Stelazine e a resposta foi surpreendentemente boa. Fiquei perplexo e, desde então, aprendi a ser modesto e humilde nas minhas crenças.

No segundo ano da minha residência iniciei a minha análise pessoal com o dr. Francis McLaughlin (1967). Foi uma experiência intensa e até hoje muito importante para a minha vida pessoal e profissional. Naquela época, essa análise lá era considerada “clássica” (Langs, 1981), com sessões individuais quatro vezes por semana, no divã, do princípio até o fim do tratamento, três anos mais tarde.

Ao terminar a minha residência, arranjei o meu primeiro emprego num grande hospital público de Maryland para deficientes mentais, que naquela época tinha 2.500 pacientes. Era uma pequena cidade com campos esportivos e escolas, e foi a minha primeira experiência num grande hospital público nos Estados Unidos. Anos mais tarde, quando pela segunda vez voltei aos Estados Unidos, fui visitar esse hospital que agora tinha apenas cem pacientes. A grande maioria havia sido transferida para residências e programas comunitários (Mosher e Burti, 1994). Eu atendi alguns desses pacientes na comunidade e até hoje não estou 


\section{$\begin{array}{lllllll}R & E & V & I & S & T & A\end{array}$ \\ LATINOAMERICANA \\ DE PSICOPATOLOGIA \\ F U N D A M N T A L \\ ano VII, n. 3, set/ 2005}

certo se essa mudança foi benéfica para todos. Muitos deles, que pertenciam antes a uma comunidade hospitalar, viviam agora em residências com três ou quatro outros pacientes e um supervisor, completamente isolados na comunidade maior.

Terminada a minha análise, montei o meu consultório particular e também fui trabalhar num hospital psiquiátrico particular de Baltimore, onde havia também uma forte influência psicanalítica (Forbusch, 1971). Havia uma enfermaria a muito longo prazo para pacientes crônicos. As outras eram para pacientes agudos que ficavam no hospital cerca de um ano ou mais porque, naquela época, os seguros médicos ainda pagavam internações longas. Outra vez a mesma coisa nesse hospital: dez pacientes, cada um para psicoterapia psicanalítica três vezes por semana, independentemente do diagnóstico. O programa era dirigido por dois psicanalistas, dr. Robert Gibson (1967) e Clarence Schulz, e era um pouco diferente do programa do Hospital Chestnut Lodge. Em vez da ênfase quase exclusiva na psicanálise individual, o tratamento era multidimensional e a psicoterapia era apenas um dos componentes de um sistema de atendimento integrado e organizado que incluía: administração clínica e comunidade terapêutica (Jones, 1968, Edelson, 1970, Cumming \& Cumming, 1962, Gunderson, Will e Mosher, 1983), medicação psiquiátrica, atividades terapêuticas (Erikson, 1976) e atendimento às famílias (Perlmutter, 1996, Satir, 1964, Zuk e Boszrmenyi-Nagy, s/d., Jackson, 1960, Lidz, 1968, Lidz, 1973).

Durante os meus sete anos de trabalho nesse hospital fazendo psicoterapia psicanalítica e sendo diretor de um setor, os medicamentos começaram a ser mais usados. Isso foi uma grande mudança na ideologia dos anos 1960 em que o remédio era visto quase como um fracasso da psicoterapia. Como todas as mudanças ideológicas, essa causou também suas casualidades. O dr. Harold Searles, por exemplo, o nosso mais importante consultor psicanalista, a quem apresentávamos pacientes uma vez por semana, pediu demissão do hospital quando percebeu que estávamos usando mais medicação do que ele achava necessário. Também o fato de a psicoterapia individual ter sido demovida para se tornar apenas um dos componentes do tratamento, causou muita estranheza aos psicanalistas envolvidos com o hospital.

Nessa época, ainda não havia muitos programas terapêuticos e residenciais na comunidade. Via de regra os pacientes tinham alta do hospital para voltar para suas famílias e para o consultório dos seus analistas e psiquiatras.

Em 1974 voltei para o Brasil, estabelecendo-me em Belo Horizonte. Logo percebi que a nossa psiquiatria, ao contrário da norte-americana daquela época, era acima de tudo biológica e sofria muito pouca influência da psicanálise, que então estava sendo introduzida pelo professor Malomar Lund Edelweis, por meio do Círculo Psicanalítico de Minas Gerais, onde então fiz a minha formação. 
Fiquei meio surpreso com as diferenças que encontrei: ausência da influência da psicanálise na psiquiatria brasileira e uma separação muito nítida entre as duas disciplinas. Também observei que a psicanálise mineira não era a psicanálise “clássica” que eu havia conhecido em Baltimore. O que lá era considerado psicoterapia aqui era chamado de psicanálise: sessões uma vez por semana, combinação de sessões individuais e de grupo, mudança de freqüência das sessões, decréscimo do número de sessões à medida que a análise avançava etc.

Junto com alguns colegas, criei em Belo Horizonte o Centro Psicoterapêutico, um hospital-dia, com as dimensões terapêuticas que eu havia aprendido nos EUA (Oliveira e Pinheiro, 1977) e também publiquei alguns trabalhos (Oliveira e Pinheiro, 1977, Pinheiro, 1983). Enfrentamos algumas resistências vindas tanto da psiquiatria quanto da psicanálise locais, mas para mim foi uma experiência muito importante estar de novo em contato com os colegas mineiros e comparar o que se fazia aqui com o que eu tinha aprendido lá. Esta experiência durou 13 anos (1974 a 1987), e enquanto isso muita coisa estava mudando nos EUA.

Quando voltei para lá, em 1987, a necessidade de conter custos, a desospitalização e o aparecimento dos planos e seguros saúde com o managed care passaram a ditar as normas para os atendimentos psiquiátricos, levando a grandes mudanças na psiquiatria e na psicanálise. Muitos tratamentos úteis foram gradativamente eliminados ou limitados, entre eles a socioterapia, comunidade terapêutica, psicoterapia individual e atividades terapêuticas, só para citar alguns. O tempo de permanência dos pacientes nos hospitais havia diminuído consideravelmente. Surgiram as Enfermarias Psiquiátricas em Hospitais Gerais e os Leitos de Crise para internações a curtíssimo prazo. Apareceu o desafio clínico do paciente de alta rotatividade com centenas de hospitalizações por ano.

Enfim, a psiquiatria americana voltou a ser essencialmente biológica (Andreasen e Black, 1990).

O movimento de desospitalização gerou inicialmente vários desastres e teve de ser interrompido para ser reiniciado mais tarde, com mais cuidado, depois de instalados programas mais abrangentes residenciais e de atendimentos comunitários, especialmente no setor público. A grande esperança era a extinção dos hospitais psiquiátricos não só para economizar dinheiro, mas também para oferecer melhores tratamentos na comunidade. Contudo, isso não ocorreu até hoje, apesar do grande avanço que tem sido feito e da dramática redução dos leitos hospitalares. Com essas mudanças, a psiquiatria dinâmica norte-americana que eu havia aprendido nos anos 1960, praticamente acabou. A psiquiatria norteamericana voltou a ser uma psiquiatria essencialmente biológica e os laboratórios passaram a ter grande influência nos congressos e até na pesquisas. Por influência dos planos e seguros saúde, os psiquiatras já não eram mais psicoterapeutas, mas sim “Revisores da Medicação” com sessões de 15 minutos. Muitas 
residências não mais ofereciam cursos em psicoterapia. Um grande número de hospitais particulares faliu sob a influência dos planos e seguros saúde. A psicanálise perdeu muito o seu prestígio, e os chefes de departamentos acadêmicos já não eram mais psicanalistas. Fiquei tão impressionado com essas mudanças que publiquei dois artigos sobre o que presenciara (Pinheiro, 1989, 1992).

Esta segunda experiência nos Estados Unidos durou 14 anos, quando retornei novamente ao Brasil (2001). Verifiquei, então, que enquanto a psicanálise nos EUA já não tinha grande influência na psiquiatria, no Brasil era agora exatamente o oposto: uma psicanálise muito presente e influente não só na psiquiatria, mas na sociedade em geral (Garcia, 2002). Encontrei também um movimento muito forte de desospitalização (Barreto, 1999), mas enquanto aqui todos ainda acreditam que os programas comunitários podem acabar com os hospitais, os EUA, depois de passarem por essa fase, estão agora tentando estudar o papel do hospital no sistema de saúde mental.

Enfim, reencontrei no Brasil uma cisão muito intensa entre a psicanálise e a psiquiatria biológica. Enquanto isso agora ocorre aqui, nos EUA o movimento caminha para uma integração entre as neurociências e a psicanálise. Alguns neurocientistas estão descobrindo que muita coisa que Freud falou está sendo confirmado pelas suas pesquisas e muitos psicanalistas estão considerando que suas intervenções terapêuticas são intervenções neurobiológicas.

Interessante e curioso que na minha primeira volta ao Brasil, em 1974, saí de uma psiquiatria psicanalítica norte-americana para encontrar aqui uma psiquiatria essencialmente biológica. Em 2001, saí de uma psiquiatria essencialmente biológica para encontrar aqui uma psiquiatria psicanalítica, pelo menos em certos setores. Vejo também no horizonte brasileiro o aparecimento da psiquiatria biológica e da psiquiatria controlada pelas empresas da saúde, como aconteceu nos EUA. Percebo que isso está crescendo, especialmente nos meios acadêmicos, e vejo que não só os remédios, mas também o ECT e até as psicocirurgias estão voltando a ter um lugar importante nas intervenções terapêuticas.

\section{Onde estamos hoje?}

Depois de todos esses anos na psiquiatria e na psicanálise nesses dois países, vi muitas coisas, tanto no campo da psiquiatria quanto no campo da psicanálise, surgirem e desaparecerem. Existem hoje mudanças importantes na psiquiatria, psicanálise e na saúde mental.

Na psiquiatria temos as novas medicações, novos exames e novas pesquisas. Existe também o movimento da desospitalização. Na psicanálise, depois de Freud 
passamos por Melanie Klein, Guntrip, Fairbain, Winnicott e Kohut. Vi Jacques Lacan aparecer nos Estados Unidos (Schneiderman, 1983; Zizek, 1991; Fink, 1995, 1997), mas quando cheguei aqui fiquei perplexo com a intensidade de sua penetração no Brasil. Na saúde mental, o progresso maior está na ênfase à cidadania plena para o doente mental e à noção de a saúde mental depender do meio sociocultural onde o cidadão se insere.

Mesmo com esses avanços, devemos sempre ter cuidado para não jogar fora o que foi aprendido antes quando aparecesse algo novo. No nosso campo, ainda tão incerto, as coisas vão e vêm, e não podemos esquecer que "vinhos velhos às vezes são servidos em garrafas novas”. Por isso, devemos evitar radicalizar e precisamos cultivar uma certa modéstia para perceber que estamos longe de saber, com certeza, os nossos diagnósticos, etiologias e tratamentos. Precisamos ter flexibilidade para estar sempre abertos a todas as evidências vindas de todas as fontes que possam nortear a nossa conduta clínica. Devemos ser capazes de considerar as muitas dimensões do nosso paciente antes de decidir qual intervenção terapêutica iremos tentar, com uma disposição de mudar de estratégia se ela não for bem-sucedida. Uma abordagem de tentativa e erro.

Penso que o futuro do nosso campo será a convergência entre as neurociências, a psiquiatria e a psicanálise, de maneira que os nossos tratamentos possam ser informados pelas três disciplinas. Afinal, elas tratam das coisas que se passam no mesmo lugar: o cérebro.

\section{Referências}

Andreasen, N.C. e Black, D. W. Introductory Textbook of Psychiatry. Washington, DC: American Psychiatric Press, 1990.

Barreto, F.P. Reforma psiquiátrica \& movimento lacaniano. Belo Horizonte: Itatiaia, 1999.

Cameron, Norman. Personality Development and Psychopathology. Boston: Houghton Mifflin, 1963.

Cumming, J. e Cumming, E. Ego \& Milieu: Theory and Practice of Environmental Therapy. Chicago: Aldine Publishing Co., 1962.

Edelson, M. The Practice of Sociotherapy. Connecticut: Yale University Press, 1970.

Erikson, Joan M. Activity, Recovery, Growth. The Communal Role of Planned Activities. New York: W.W. Norton \& Co., 1976.

Fink, B. The Lacanian Subject: Between Language and Jouissance. Princeton, New Jersey: Princeton Universities Press, 1995. 
A Clinical Introduction to Lacanian Psychoanalysis. Cambridge, Mass: Harvard Universities Press, 1997.

Forbusch, B. The Sheppard \& Enoch Pratt Hospital: 1853-1970, A History. Philadelphia \& Toronto: J.B. Lippincott Co., 1971.

Fromm-Reichmann, F. Principles of Intensive Psychotherapy. Chicago: University of Chicago Press, 1950.

Garcia, C. Psicanálise, psicologia, psiquiatria e saúde mental. Belo Horizonte: Interfaces, Ophicina de Arte e Prosa, 2002.

Gibson, R. W. Crosscurrents in Psychiatry \& Psychoanalysis. Philadelphia: J.B. Lippincot Co., 1967.

Green, H. (or Greenberg, J.). I Never Promised you a Rose Garden. New York: Holt, Rinehart \& Winston, 1964.

Gunderson, J.G., Will, O. E Mosher, L. Principles and Practices of Milieu Therapy. New York: Aronson, 1983.

Horstein, G.A. To Redeem one Person is to Redeem the Word: The Life of Frieda Fromm-Reichmann. New York: The Free Press, 2000.

Psychoanalysis and Psychotherapy. Chicago: The University of Chicago Press, 1959.

JACKson, D.D. The etiology of Schizophrenia. New York: Basic Books, 1960.

Jones, M. Social Psychiatry in Practice: The idea of the Therapeutic Community. Baltimore: Penguin Books, 1968.

Kaplan, H.I. Comprehensive Textbook of Psychiatry. Baltimore: The Williams \& Wilkins Co., 1967.

Langs, Robert. The Therapeutic Relationship and deviations in technique. In: Langs, R. (org.). Classics in Psychoanalytic Technique. New York: Jason Aronson, 1981.

Lewin, R. e Schulz, C. Losing and Fusing: Borderline Transitional Object and Self Relations. Northvale: Jason Aronson, 1992.

Lidz, Theodore. The Person throughout the Life Cycle. New York: Basic Books, 1968.

The Origin \& Treatment of Schizophrenic Disorders. New York: Basic Books, 1973.

McLaughlin, Francis. On the concept of health. In: Gibson, R.W. (org.). Crosscurrents in Psychiatry and Psychoanalysis. Philadelphia and Toronto: J.B. Lippincott Co., 1967. Mosher, L. e Burti, L. Community Mental Health: a Practical Guide. New York: W.W. \& Norton Co., 1994.

Oliveira, D.T. e Pinheiro, M.V. Hospital-dia como uma comunidade terapêutica. Psicologia Clínica e Psicoterapia, n. 2, p. 15-9, 1977. 
Perlmutter, R.A. A Family Approach to Psychiatric Disorders. Washington DC: American Psychiatric Press, 1996.

Pinheiro, M.V. Hospital Sheppard Pratt: um hospital psicoterapêutico. Estudos de Psicanálise, n. 6, p. 85-90, 1973.

O tratamento do Episódio Esquizofrênico Agudo. Revista da Associação Médica de Minas Gerais, n. 34, p. 10-5, 1983.

U.S.A. Thirteen Years Later. Psychiatry. New York: Guilford Publications, 1989. The selling of clinical psychiatry in America. Hospital and Community Psychiatry, n. 43, p. 102-4, 1992.

SatiR, V. Conjoint Family Therapy. Palo Alto, California: Science and Behavior Books, 1964.

Schneiderman, S. Jacques Lacan: The Death of an Intelectual Hero. Cambridge, Mass: Harvard Universities Press,1983.

Schulz, C. e Kilgalen, R. K. Case Studies in Schizophrenia. New York: Basic Books, 1969.

Searles, H. Collected Papers on Schizophrenia and Related Subjects. New York: International Universities Press, 1965.

Sullivan, H.S. The Psychiatric Interview. New York: W.W. Norton \& Co., 1954.

The Interpersonal Theory of Psychiatry. New York: W. W. Norton \& Co., 1953.

Will, Otto Allen Jr. Schizophrenia: psychological treatment. In: Freedman, A.M. e Zuk, G.H. e Boszrmenyi-Nagy, I. Family Therapy and Disturbed Families. Palo Alto, California: Science and Behavior Books, 1967.

ZızeK, S. Looking Awry: An Introduction to Jacques Lacan Through Popular Culture. Cambridge, Mass: The MIT Press, 1991.

\section{Resumos}

El autor trabajó intensamente durante muchos años como psiquiatra y psicoanalista en Brasil y los Estados Unidos, en un período de cuarenta y cinco años. Relata sus experiências en esos dos países, apunta semejanzas y diferencias en el trabajo clínico durante esos años, así como las tendências en diferentes épocas. Concluye haciendo una previsión sobre lo futuro del trabajo clínico en los EEUU y Brasil.

Palabras claves: Brasil, Estados Unidos, trabajo clínico, psiquiatría 
L'auteur a travaillé en tant que psychiatre et psychanalyste au Brésil et aux Etats Unis pendant environ quarante cinq années.Fort de cette longue expérience, il nous livre ses réflexions sur son travail clinique dans les deux pays, insistant sur leurs ressemblances, leurs différences et les changements qu'il a notés tout au long de cette période. En guise de conclusion, il émet certaines hypothèses sur l'avenir de la clinique psychiatrique et psychanalytique aussi bien aux EEUU qu'au Brésil.

Mots clés: Brésil, Etats Unis, travail clinique, psychiatrie

The author has worked extensively as a psychiatrist and psychoanalyst in Brazil and United States in a period of forty-five years. He writes on his experiences in both countries, highlighting similarities and differences in the clinical work during those years. He brings the reader's attention for tendencies in each country in different times. He concludes by making a prediction on the future of the clinical work in both countries.

Key words: Brazil, United States, clinical work, psychiatry

Versão inicial recebida em junho de 2005

Versão revisada recebida em junho de 2005 